\title{
Dinâmica espaço-temporal da expansão urbana brasileira a partir do uso de sensoriamento remoto - uma revisão
}

Space-time dynamics of brazilian urban expansion from the use of remote Sensing - a review

Dinámica espacio-tiempo de la expansión urbana brasileña a partir del uso de la teledetección - una revisión

José Erivaldo da Silva

ORCID: https://orcid.org/0000-0001-6682-852X Universidade Federal de Campina Grande, Brasil e-mail: jerysilva85@gmail.com

Andréia Motta Coelho Crispim ORCID: https://orcid.org/0000-0002-8813-5094 Universidade Federal de Campina Grande, Brasil e-mail: andrea.mottacrispim@gmail.com

Janaína Barbosa da Silva

ORCID: https://orcid.org/0000-0001-6366-2165 Universidade Federal de Campina Grande, Brasil e-mail: janainamov@yahoo.com.br

\begin{abstract}
Resumo
O Sensoriamento Remoto (SR) é uma ferramenta de suma importância para o estudo do uso e ocupação do solo no Brasil. A produção científica com essa temática vem apresentando resultados cada vez mais eficientes tanto em números quanto em qualidade dos trabalhos. Assim, objetivou-se identificar o uso do SR aplicado ao estudo e análise de áreas urbanas no Brasil, para isto foram identificados os satélites utilizados nas pesquisas; os métodos e as técnicas para os estudos das áreas urbanas e por fim, identificar as Regiões brasileiras que mais utilizam dados de SR para estudos de suas áreas urbanas. Para tanto, utilizou-se um protocolo de pesquisa, sendo empregados artigos que apresentavam uma classificação hierárquica do uso do solo urbano, para isto foram pesquisados artigos indexados no período de 1972 a 2020 em três bases eletrônicas: ScienceDirect, Plataforma Capes/ CAFÉ e o Google acadêmico. Este procedimento resultou em283artigos, reduzidos para 54 artigos, que passaram a compor a amostra desta pesquisa. Como resultado por Regiões tem-se por ordem decrescente o Sudeste, Sul, Nordeste, Centro Oeste e Norte. Os satélites mais utilizados foram respectivamente.
\end{abstract}

Palavras-chave: Uso e ocupação do solo; Espaços urbanos; Planejamento urbano.

\begin{abstract}
The use of remote sensing has been a tool of paramount importance for the study of land use and occupation, in Brazil, scientific production with this theme has been presenting increasingly efficient results both in numbers and in the quality of the works, so this study is based on a bibliographic review of remote sensing in Brazilian urban areas, thus having the main objective of this study was to identify the use of remote sensing applied to the study and analysis of urban areas in Brazil, for this we identified the satellites used in the researches; the methods and techniques for the studies of urban areas and list the Brazilian regions that most use remote sensing data for studies of urban areas. For this purpose, we used a research protocol, and articles were used that presented a hierarchical classification of urban land use, for this we searched indexed articles in the period from 1972 to 2020 on three electronic bases: ScienceDirect, the Capes/CAFÉ platform and Google Scholar. This procedure resulted in the amount of 283 results computed in the selected search engines, where 54 (Fifty-five) articles were selected that began to compute the sample for the study in question, as a result by regions the analysis of the sample demonstrates that in quantitative terms the Brazilian regions appear in the following order: Southeast, South, Northeast, Midwest and North, and in both there is the use of remote sensing in urban planning, given the proportionalities of importance depending on the degree of understanding of the importance of this tool by decision makers.
\end{abstract}

Keywords: Use and occupation of urban land; Urban spaces; Urban planning.

\section{Resumen}

La teledetección (RS) es una herramienta muy importante para el estudio del uso y la ocupación de la tierra en Brasil. La producción científica con esta temática ha presentado resultados cada vez más eficientes tanto en número como en calidad de los trabajos. Así, el objetivo fue identificar el uso de SR aplicado al estudio y análisis de áreas urbanas en Brasil, para ello identificamos los satélites utilizados en las investigaciones; los métodos y técnicas para los estudios de áreas urbanas y, finalmente, identificar las regiones brasileñas que más utilizan datos de RS para estudios de sus áreas urbanas. Para ello, se utilizó un protocolo de investigación, utilizando artículos que presentaban una clasificación jerárquica del uso del suelo 
urbano, para ello se buscaron artículos indexados en el periodo de 1972 a 2020 en tres bases electrónicas: ScienceDirect, Capes/CAFÉ Platform y Google academic. Este procedimiento dio como resultado 283 artículos, reducidos a 54 a consa componer la muestra de esta investigación. Como resultado de las regiones, el sureste, el sur, el noreste, el medio oeste y el norte están en orden descendente. Los satélites más utilizados fueron respectivamente.

Palabras clave: Uso y ocupación de la tierra; Espacios urbanos; Urbanismo.

\section{Introdução}

O Sensoriamento Remoto enquanto técnica surgiu logo após a invenção da fotografia, quando se tornou possível o registro de imagens sub-orbitais, tornando-se de grande importância estratégica em tempos de guerra para o reconhecimento do território inimigo e a partir de 1972 as imagens orbitais são uma realidade a partir do lançamento de satélites. Portanto, pode-se dizer que foi a partir da Primeira Guerra Mundial que essa técnica começou a ser aperfeiçoada (Florenzano, 2002).

Segundo (Barros, 2005), o Sensoriamento Remoto é a técnica de obtenção de informações acerca de um objeto, área ou fenômeno localizado na Terra, sem que haja contato físico. Sendo ele uma técnica alternativa muito eficiente para avaliação do crescimento urbano, devido à possibilidade de se obter imagens de extensas áreas da superfície terrestre, com isso permitindo a definição dos limites e monitoramento urbano, bem como possíveis problemas ambientais decorrentes do processo de expansão urbana, (Jensen, 2009). A utilização desses dados torna-se uma ferramenta eficiente no planejamento urbano.

As análises da dinâmica de alterações no território para o planejamento urbano contam com os avanços tecnológicos nas áreas espaciais, principalmente na área de sensoriamento remoto. Na literatura específica, cada vez mais as pesquisas se valem de geotecnologias para análise em ambientes urbanos, ressaltando-se que a distribuição dos usos do solo sobre a área urbana determina a localização das atividades humanas (Haas et al., 2018; Mushorea et al., 2017; Amani-Beni et al.;2018; Zhou et al., 2019); especialmente em espaços urbanos, nos quais os estudos do sensoriamento remoto tende a eficiência.

Os estudos de (Forster, 1994; Fuckner, 2006; Almeida, 2010; \& xianmig, et al, 2021), sobre o Sensoriamento Remoto (SR) e os espaços urbanos se completam. Quando ressaltamos SR como um dos maiores avanços já produzido pela ciência e tecnologia no que tange ao estudo da superfície terrestre, quando da sua eficiência para avaliar o processo de crescimento do espaço urbano, em detrimento do avanço do desmatamento, dos problemas ambientais decorrentes desse processo de expansão, entre outros, gerando significativos problemas ambientais.

Segundo (Portis, et. al, 2020), ressaltam que imagens de sensoriamento remoto configuram instrumentos valiosos de coleta de dados para análise do território, especialmente para o planejamento, a mobilidade, a acessibilidade e a sustentabilidade.

As áreas urbanas são fisicamente formadas por uma grande diversidade de materiais (superfícies de concreto, asfalto, telhados de vários materiais, solo, vegetação, arbórea, grama, água entre outros), com formas, alturas e arranjos, também variados (Jensen, 2009; Almeida, 2010). Segundo (Costa \& Alves, 2005; \& Zoangping et al, 2013), as áreas urbanas não podem ser caracterizadas por um comportamento espectral padrão, diferente do que acontece com vegetação, os corpos aquáticos e os solos, esses são superfícies relativamente homogêneas em termos de cobertura, enquanto, nas áreas urbanas, o que prevalece é a heterogeneidade das coberturas das áreas construídas, fatores que tendem ao sinistro, uma vez que a heterogeneidade dos espaços urbanos eleva os riscos à biodiversidade.

Pode-se dizer que são notórios os riscos e prejuízos causados pelo crescimento desenfreado e ocupação desordenada das áreas urbanas, com isto acarretando no desaparecimento da biodiversidade vegetal e animal, dos recursos hídricos, e a contaminação do meio físico são algumas das consequências da expansão urbana ao longo dos anos (Aragão et al., 2017).

Os aspectos ligados a urbanização como a localização do sítio urbano, limite da área urbana, expansão e o processo de conurbação, são facilmente identificados em imagens orbitais (Oranquine, 2020); (Xianmin, 2021). Ademais, ainda por meio 
de imagens de satélite, é possível distinguir cidades planejadas, de uma cidade que nasceu e se desenvolveu espontaneamente, pois o ser humano transforma os espaços por diversos meios, como a derrubada de árvores, implantação de pastagens, construções de estradas, portos, cidades inteiras etc. (Monteiro \& Mendonça, 2003).

As mudanças ambientais decorrentes das ações antrópicas como resposta a degradação ambiental têm refletido em alterações no clima em escalas global ao local (Miranda, 2018). Alterações climáticas são tão evidentes que, na definição do termo "clima urbano", muitos trabalhos têm dado destaque ao efeito observado nas cidades ao invés de evidenciar a atmosfera. Sendo assim, o clima urbano é um sistema complexo, adaptativo e aberto que, ao receber energia do ambiente maior no qual se insere, a transforma substancialmente a ponto de gerar uma produção exportada ao ambiente (Tavares \& Prochnow, 1997; Monteiro \& Mendonça, 2003, IPCC, 2021).

Desse modo, (Miranda, 2018; Souza, 2001 \& IPCC, 2021), destacam alguns dos impactos ambientais advindos de atividades antropogênicas, como: a poluição atmosférica e das águas, produção de lixo, alterações no microclima (ilhas de calor), a destruição do solo e da cobertura vegetal, são causadas por chuvas torrenciais e escorregamento de encostas.

Desse modo, as imagens obtidas por sensores remotos contribuem na identificação desses diferentes usos do espaço terrestre, nos quais podemos a partir da escala de municípios identificar e classificar os espaços em urbano e rural, além disso, o aspecto multitemporal dessas imagens permite acompanhar as transformações do espaço ao longo do tempo. Em suma, $\boldsymbol{o}$ objetivo principal deste artigo de revisão foi identificar o uso do Sensoriamento Remoto aplicado ao estudo e análise de áreas urbanas no Brasil, no período de 1972 a 2020, mais precisamente: Averiguar quais satélites são mais utilizados nas pesquisas; identificar os métodos e as técnicas para os estudos das áreas urbanas; estabelecer uma hierarquia das Regiões brasileiras a partir do uso de dados de Sensoriamento Remoto para estudos das áreas urbanas.

Devido à magnitude do tema, existiu uma dificuldade em separar artigos que realmente apresentassem relevância para essa pesquisa, assim, buscou-se apenas produções relacionadas à identificação de estudos que se relacionassem com estudos dos espaços urbanos vinculados ao uso e ocupação do solo e que esses estudos tivessem sido realizados a partir da utilização de sensoriamento remoto.

\section{Metodologia}

A presente pesquisa enquadra-se em uma pesquisa qualitativa, pois trata-se de uma análise da produção cientifica nacional e/ou internacional realizadas a partir de estudos de sensoriamento remoto voltados ao uso e ocupação dos solos urbanos, a análise foi realizada tomando por base características da indução que para (Pereira, et. al. 2018); a indução por si só parte do particular, deixando a generalização como um produto posterior, compra-se a necessidade a necessidade de conhecer o produto como um todo (Gil, 2008), desse modo, a compreensão da ocupação dos solos urbanos necessitam de ser entendido como uma essencialidade ao gerenciamento de políticas públicas, isto posto, pela compreensão que a qualidade de vida e a sustentabilidade dos espaços urbanos, de modo geral agrega-se ao processo indutivo da análise dos discursos e tomada de decisões, especialmente ao processo de produção cientifica (Gil, 2008).

Para atingir o objetivo principal proposto, foi definida uma seleção de artigos científicos com especificações para "mapeamento do uso dos solos em áreas urbanas nos municípios brasileiros", por Regiões geográficas na perspectiva de um mapeamento gradual dos solos urbanos, com o propósito de hierarquizar as regiões por número de produções cientificas com uso de sensoriamento remoto para análise e estudos de solos urbanos.

Para a realização da pesquisa utilizou-se como palavras chave: sensoriamento remoto, uso e ocupação do solo urbano, espaços urbanos e planejamento urbano, a partir de dados de sensoriamento remoto, no intuito de montagem de um banco de dados que permitisse mapear as regiões brasileiras pelo número de produções cientificas que de algum modo utilizassem dados de sensoriamento remoto para estudos do uso e ocupação dos solos urbanos. 
Para montagem do banco de dados foram utilizadas ferramentas como: os bancos de dados da ScienceDirect, a plataforma Capes/CAFÉ e o Google Acadêmico, com uso de computadores conectados à internet, o levantamento dos artigos e a triagem ocorrem em três etapas devidamente descritas a seguir, foram utilizados também livros que tratassem do assunto como (Jessen, 2009), teses de doutorado, dissertação de mestrado e artigos científicos e outros materiais como caderno para anotações entre outros que se fez necessário, analisando especialmente os discursos e a qualidade desses para o processo de tomada de decisão.

A análise dos discursos ocorreu por testes comparativos resultando em uma amostra aleatória, obtida a partir da análise dos objetivos e da metodologia dos artigos selecionados para compor a amostra desse trabalho, enquadrando esta em uma pesquisa qualitativa que para (Pereira, et. al. 2018, p.67): "os métodos qualitativos são aqueles nos quais é importante a interpretação por parte do pesquisador", é um tipo de pesquisa que requer atenção, posto que a análise dos dados segue métodos indutivos, isto porque "a preocupação do processo é predominantemente em relação a do produto (Pereira, et. al., 2018, p.667)", de sorte que o método indutivo segue características da observação de casos específicos e observáveis, parte do particular e não deve ser buscadas particularidades, (Gil, 2008 \& Aquino, 2010).

A Plataforma Capes a partir da base principal da Web of Sciencee o ScienceDirect foram utilizados para otimizar o número de artigos sobre o tema, aumentando o alcance das palavras-chave, o Google Acadêmico serviu de parâmetro para comparar a quantidade de materiais disponibilizado ao público de modo geral, visto que os dois primeiros são utilizados basicamente por pesquisadores.

Os idiomas utilizados foram o Português, o Inglês e o Espanhol, o intervalo de tempo foi de 48 anos de 1972 a 2020. Utilizou-se o string de busca com os operadores "OR" ou "END", possibilitando ao sistema de busca otimizar a pesquisa.

Os artigos duplicados e/ou que não atendessem aos critérios de corte foram desconsiderados, para os duplicados, considerou-se apenas um e desse modo os que estivessem na ScienceDirect. Todos os dados foram trabalhados e transformados em planilhas utilizando o programa do Excel.

Com o objetivo de tornar viável e coerente a pesquisa e com o intuito de hierarquizar as Regiões com maior número de publicações acerca do Sensoriamento Remoto aplicado ao uso de solos urbanos, adotou-se um modelo hierárquico eliminando as produções que de algum modo não se adéquem aos objetivos. O pressuposto dessa metodologia é alcançar o menor nível de detalhamento para ser analisado, no caso desta, foram às produções cientificas que de algum modo tratavam também da ocupação de solos em áreas rurais/urbanas e/ou que apresentasse outro viés que não a ocupação e uso do solo urbano.

Para tanto, foi necessário selecionar os artigos que classificassem o uso do solo urbano com base em detalhamento, no intuito de se obter uma representação cartográfica quali-quantitativa da ocupação e constituição das cidades, assim sendo, esse trabalho utilizou artigos que apresentaram uma classificação hierárquica do uso do solo urbano nas Regiões geográfica brasileiras (Anderson, et al, 1976), sugerem a compreensão do espaço urbano a partir da forma de ocupação do uso do solo, que grosso modo se dar por meio de invasões e "favelamento".

Tal qual (Anderson, et al. 1976), o sistema europeu de ocupação dos solos urbanos dar-se a partir dos sistemas de níveis, o CORINE land Cover. Seguindo esse mesmo raciocínio (Henriques, 2008), classifica por níveis as unidades das classes residenciais, como um indicador a ser considerado ao se estudar o uso do solo urbano. Desse modo, para efeito de facilitação da análise e, considerando os critérios acima mencionados pelos autores, eliminou-se no processo de seleção dos artigos todos aqueles que de algum modo apresentava confusão quanto a classificação do uso do solo urbano por níveis de ocupação e/ou aqueles que além dos espaços urbanos também faziam alusões a ocupação de áreas rurais.

A classificação de cobertura e uso do solo urbano é uma tendência em trabalhos recentes, é a utilização da chamada 'análise de imagem orientada a objeto ('Object-Based-Analysis em inglês ou OBIA'), que pressupõe o uso de redes semânticas 
e múltiplos níveis de classificação interligados, possibilitando simular a visão contextual de um intérprete humano (Panizza \& Fonseca, 2011).

A análise da ocupação e uso dos solos urbanos a partir de níveis com dados de Sensoriamento Remoto em artigos científicos foi critério para seleção de trabalhos a serem utilizados nesse estudo. A seleção foi realizada em três etapas, a saber: a primeira foi a construção de um banco de dados,onde a seleção deu-se pelos títulos e uma rápida leitura nos objetivos contidos nos resumos, o critério básico para essa primeira seleção estava na exigência que os artigos apresentassem relação com estudo de "uso e ocupação do solo em áreas urbanas realizado a partir de Sensoriamento Remoto". O que resultou em duzentos e oitenta e três artigos (283) que tratavam de estudos de áreas urbanas.

$\mathrm{Na}$ segunda etapa, foi realizada uma leitura mais acurada dos artigos considerando os critérios acima mencionados e, acrescentando a relação com alguma Região geográfica do Brasil e/ou serem artigos voltados a estudos em território Nacional, mesmo sem especificar a Região. Na terceira e última, além dos critérios de corte acima mencionados, acrescentou-se nesse último ponto a necessidade de os artigos selecionados terem uma metodologia clara de classificação do uso dos solos urbanos por níveis hierárquicos, também se acrescentou a esta etapa o critério de o artigo ser de estudos de ocupação e uso de solos urbanos sem nenhum vínculo de uso e ocupação de solos de zonas rurais.

Para operacionalizar o estudo e viabilizar a pesquisa, elaborou-se um protocolo de pesquisa no intuito de delineamento de todo o processo da pesquisa, utilizado na base de dados da plataforma CAPES/CAFÉ, ScienceDirect e Google acadêmico, ver Figura 1.

Figura 1 - Organograma dos Procedimento e etapas de pesquisa.

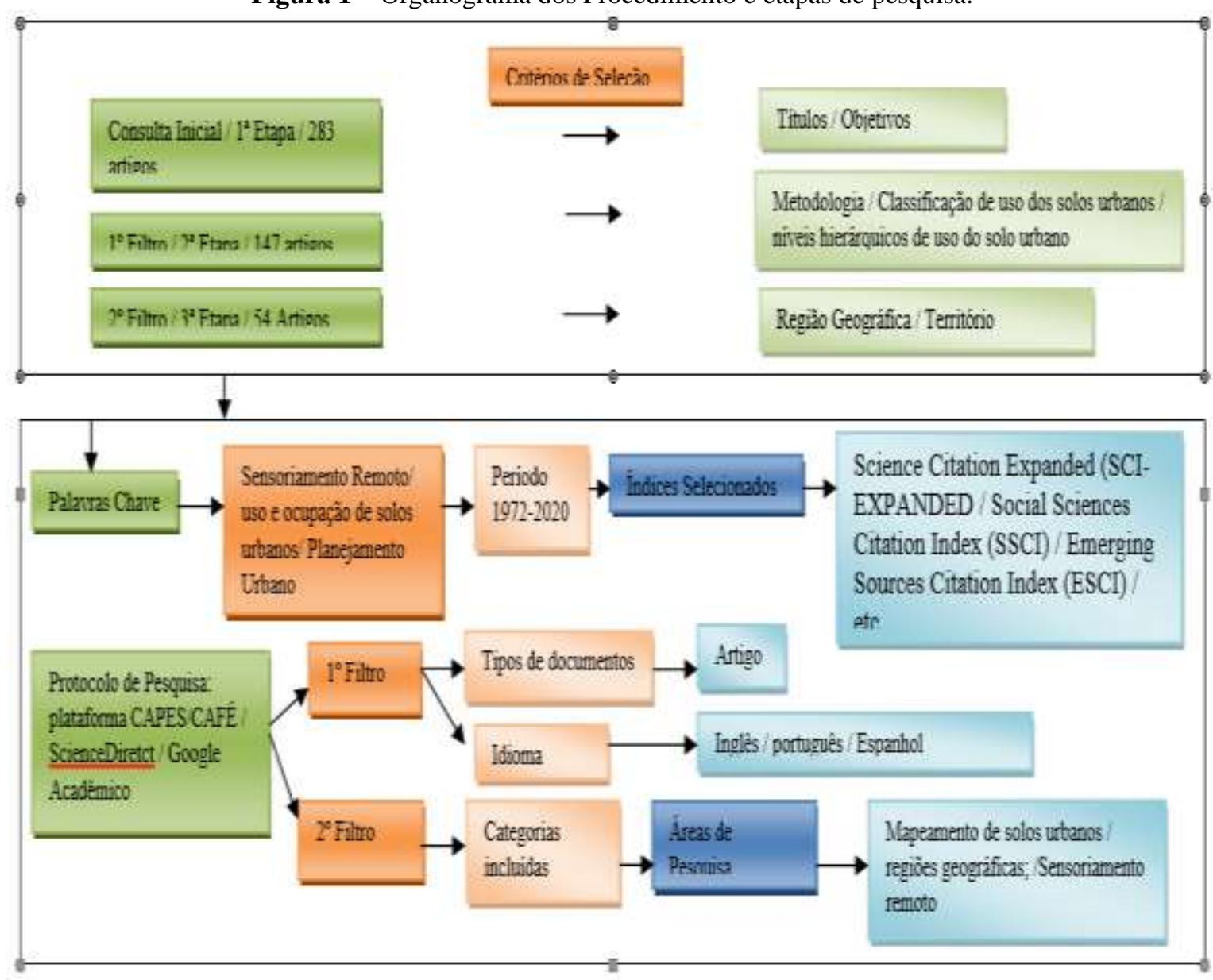

Fonte: Autores (2021). 
Desse modo, a amostra se constituiu da seguinte forma: na primeira etapa selecionou-se duzentos e oitenta e três (283) artigos, após a segunda triagem esse número passou paracento e quarenta e sete (147) artigos e, após a terceira etapa foram selecionados Cinquenta e Quatro (54) artigos que passaram a compor a amostra para o estudo em questão (Gráfico 1).

Gráfico 1 - Número de artigos por etapa.

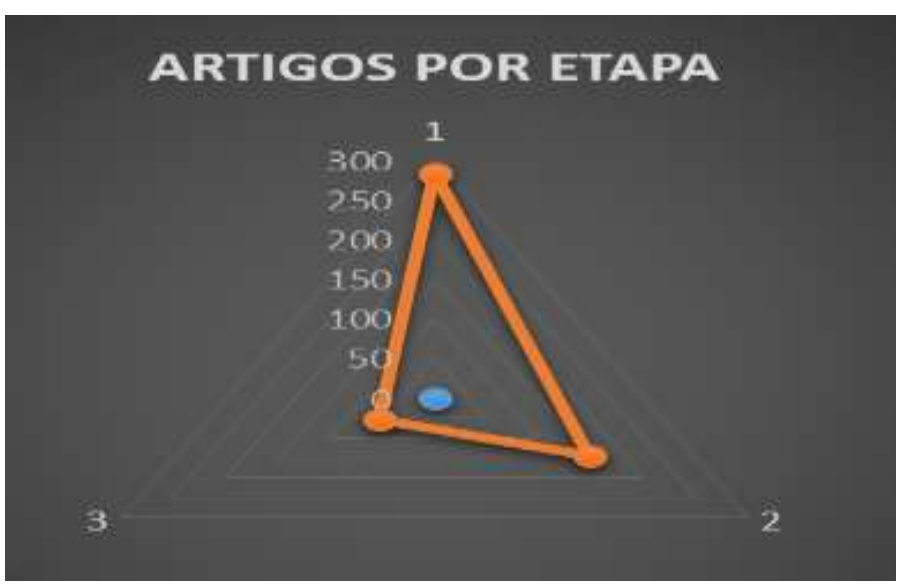

Fonte: Autores (2021).

\section{Resultados e Discussões}

Após análise das produções científicas que compõem a amostra para a realização desse estudo, evidenciou-se que no Brasil, os estudos realizados para a compreensão e análise do uso e ocupação dos solos urbanos, utilizando-se dados de Sensoriamento Remoto ocorreram basicamente a partir da utilização de imagens dos Landsat 5 (TM) e 7 (ETM+), Ikonos e Quick Bird.

Dos 54 trabalhos considerados nesse estudo, 20 são produções dos últimos cinco anos, ou seja, 37,03\%. Enquanto 34 ou 62,96\% têm mais de cinco anos de publicados. Dos 20 trabalhos dos últimos cinco anos, 10 utilizaram imagens do Landsat7 correspondendo a $18,51 \%$, cinco utilizaram imagens do Landsat5, correspondendo a 9,25\%, três utilizaram imagens do Ikonos correspondendo a 5,55\% e dois do Quick Bird correspondendo a 3,70\%. Com relação às produções anteriores a esse período, tem-se 21 trabalhos com utilização de imagens de Landsat5 correspondendo a 38,88\%, seis utilizando imagens do Landsat7 correspondendo a $11,11 \%$, três do Ikonos correspondendo a 5,55\% e quatro usaram imagens do Quick Bird, correspondendo a 7,07\% da amostra pesquisada (Tabela 1, Gráficos 2 e 3).

Tabela 1 - Utilização de bandas de satélites nos trabalhos estudados.

\begin{tabular}{|c|c|c|c|c|c|c|c|c|c|c|c|c|c|c|c|c|}
\hline \multicolumn{9}{|c|}{ Publicações dos últimos cinco anos } & \multicolumn{8}{|c|}{ Publicações anteriores aos últimos cinco anos } \\
\hline \multicolumn{2}{|c|}{$\begin{array}{l}\text { Landsat5 } \\
\text { TM }\end{array}$} & \multicolumn{2}{|c|}{$\begin{array}{l}\text { Landsat7 } \\
\text { ETM+ }\end{array}$} & \multicolumn{2}{|c|}{ IKONOS } & \multicolumn{2}{|c|}{$\begin{array}{l}\text { QUICK } \\
\text { BIRD }\end{array}$} & \multirow{2}{*}{$\begin{array}{l}\text { To } \\
\text { tal } \\
N^{\circ}\end{array}$} & \multicolumn{2}{|c|}{$\begin{array}{l}\text { Landsat5 } \\
\text { TM } \\
\end{array}$} & \multicolumn{2}{|c|}{$\begin{array}{l}\text { Landsat7 } \\
\text { ETM+ }\end{array}$} & \multicolumn{2}{|c|}{ IKONOS } & \multicolumn{2}{|c|}{$\begin{array}{l}\text { QUICK } \\
\text { BIRD } \\
\end{array}$} \\
\hline $\mathrm{N}^{\mathrm{o}}$ & $\%$ & $\mathrm{~N}^{\mathrm{o}}$ & $\%$ & $\mathrm{~N}^{\mathrm{o}}$ & $\%$ & $\mathrm{~N}^{\mathrm{o}}$ & $\%$ & & $\mathrm{~N}^{\mathrm{o}}$ & $\%$ & $\mathrm{~N}^{\mathrm{o}}$ & $\%$ & $\mathrm{~N}^{\mathrm{o}}$ & $\%$ & $\mathrm{~N}^{\mathrm{o}}$ & $\%$ \\
\hline 5 & 9,25 & 10 & 18,51 & 3 & 5,55 & 2 & 3,70 & 20 & 21 & 38,88 & 6 & 11,11 & 3 & 5,55 & 4 & 7,40 \\
\hline
\end{tabular}

Fonte: Dados da pesquisa (2021). 
Gráfico 2 - Artigos analisados por período de publicação.

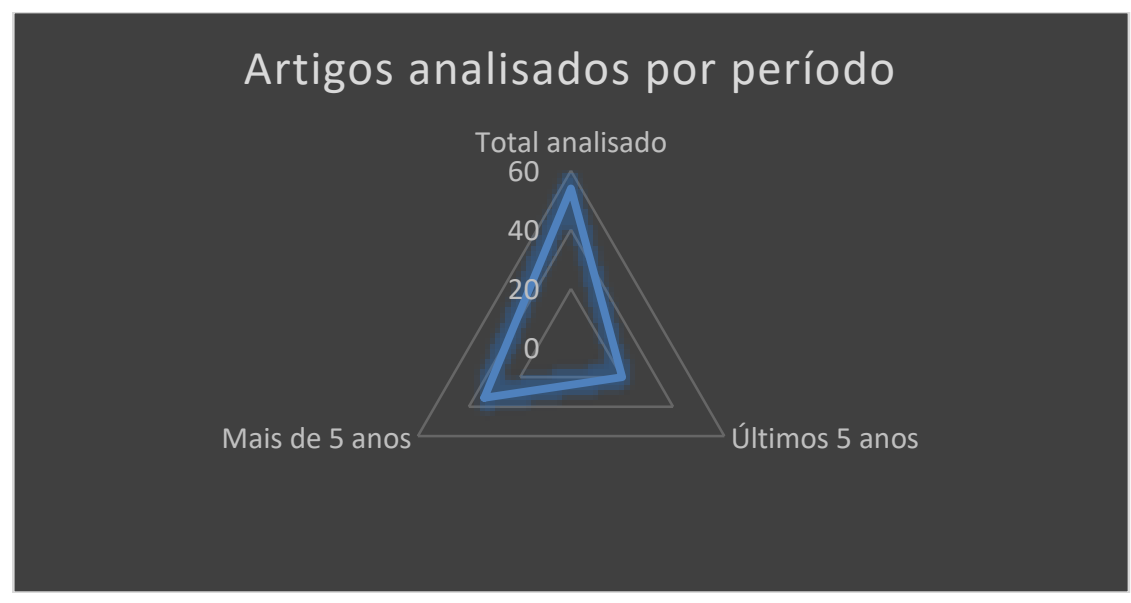

Fonte: Autores (2021).

Gráfico 3 - Satélites utilizados para estudo do solo urbano.

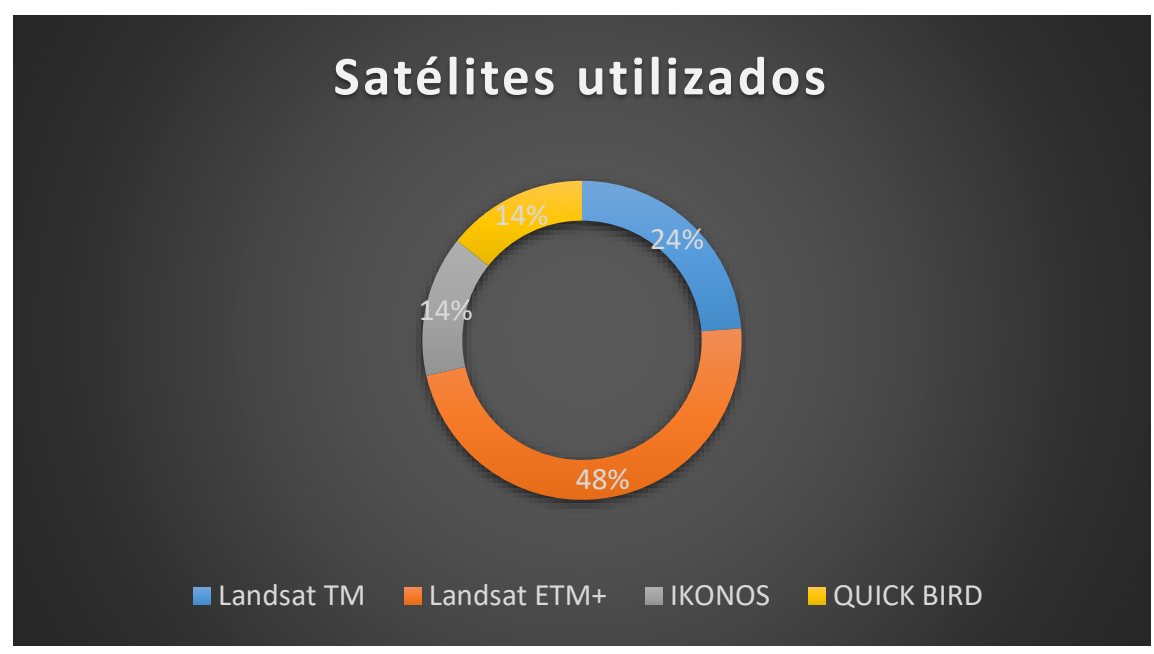

Fonte: Autores (2021).

Como técnica, a principal ferramenta foi à delimitação de pontos de ocupação por mapeamento utilizando dados de imagem do Google Earth, esses formaram-se a partir da caracterização física, vinculado quase sempre aos elementos de interpretação visual, (Panizza e Fonseca, 2011; Anderson, 1982) cor, padrão, textura, forma, localização e tamanho (Fitz, 2008).

Todos os trabalhos que foram utilizados no planejamento urbano, cujo método de classificação ocorreu de forma semiautomática, possivelmente pela possibilidade de ajustamento de distribuição de áreas a partir do interesse dos planejadores, principalmente políticos ou grupos políticos, que de alguma forma reestruturavam a área urbana dos municípios que representavam, reforçando a ideia em seus estudos quando mencionam possíveis caminhos para solucionar problemas de ocupação de solos urbanos a partir dos ajustamentos políticos dados por seus representantes para solucionar problemas como o surgimento de áreas de "afavelamento" (EEA, 1990; Castro, 2000; Costa, 2005; Fuckner, 2006 \& Leite e Brito, 2011).

Dos trabalhos publicados nos últimos cinco anos, (9) nove foram utilizados para o planejamento urbano representando 45\%, enquanto em (11) onze, não há indícios de utilização de seus dados para tal fim, representando 55\%. Dos trabalhos com mais de cinco anos de publicação apenas (7) sete apresentam resultados para o uso no planejamento urbano o que representa 20,58\% e (27) vinte e sete trabalhos não foram utilizados para o planejamento urbano representando 79,41\% (Tabela 2, Gráfico 4). Logo, o planejamento de modo geral ocorreu como dito anteriormente a depender da vontade do grupo político e do 
conhecimento que estes detêm da importância das tecnologias e dos estudos acadêmicos quando utilizados para benefício da sociedade, assim como da visão de futuro que estes grupos dispõem para o futuro dos espaços urbanos.

Tabela 2 - Número de trabalhos que foram utilizados para o planejamento urbano.

\begin{tabular}{l|c|c|l|l|c|c}
\hline \multicolumn{2}{l|}{ Publicações com até cinco anos } & \multicolumn{4}{l}{ Publicações com mais de cinco anos } \\
\hline $\begin{array}{l}\text { Trabalhos utilizados para o } \\
\text { planejamento Urbano }\end{array}$ & $\mathrm{N}^{\mathbf{0}}$ & $\%$ & $\begin{array}{l}\text { Trabalhos utilizados para o } \\
\text { planejamento Urbano }\end{array}$ & $\mathrm{N}^{\mathbf{o}}$ & $\%$ \\
\hline Sim & 9 & 45 & Sim & 7 & 20,58 \\
\hline Não & 11 & 55 & Não & 27 & 79,41 \\
\hline Total & 20 & 100 & & 34 & 99,99 \\
\hline
\end{tabular}

Fonte: Dados da pesquisa (2021).

Gráfico 4 - Uso para o planejamento urbano.

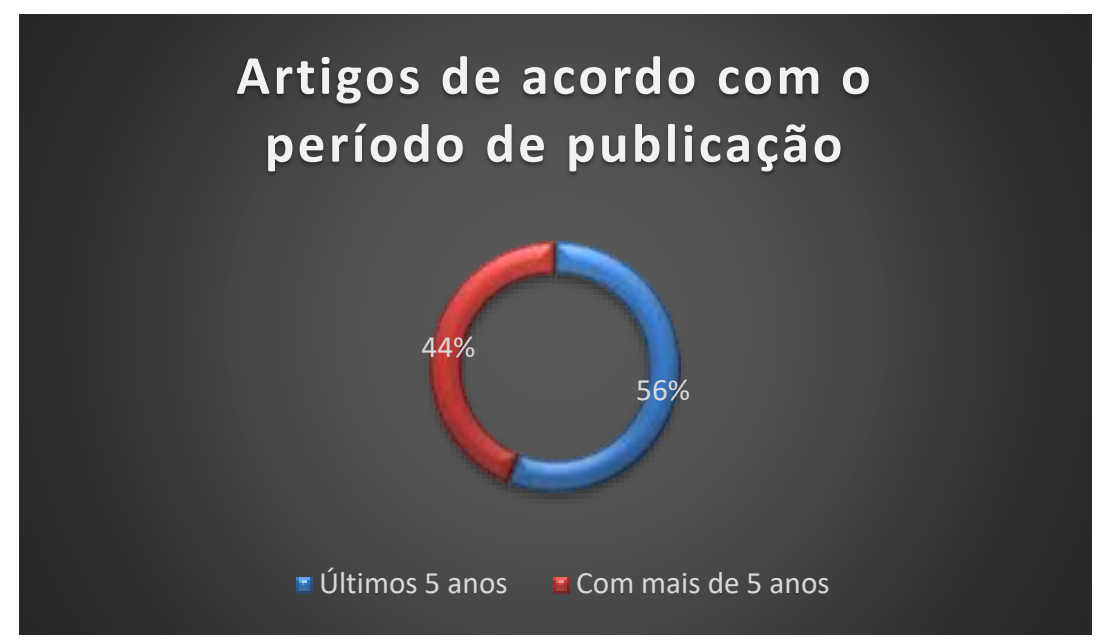

Fonte: Autores (2021).

Independente do uso ou não dos dados técnicos disponíveis nos trabalhos para o planejamento urbano, os municípios de grande e médio porte lideram na utilização desse recurso para planejar ou analisar a área urbana. Os municípios de pequeno porte não aparecem como utilizadores desses recursos. (Quadro 1). 
Quadro 1 - Caracterização dos tipos de uso e ocupação dos solos urbanos.

\begin{tabular}{|c|c|c|c|c|c|c|}
\hline \multicolumn{3}{|c|}{ Tipo de ocupação $100 \%$ urbana } & \multirow{2}{*}{\multicolumn{4}{|c|}{ Classe de uso e ocupação identificada }} \\
\hline \multirow{2}{*}{$\begin{array}{ll}\text { Porte } & \text { do } \\
\text { Município } & \end{array}$} & \multicolumn{2}{|c|}{$\begin{array}{l}\text { Uso dos dados } \\
\text { planejamento urbano }\end{array}$} & & & & \\
\hline & Sim & Não & & & Sim & Não \\
\hline \multirow{5}{*}{ Médio } & \multirow{5}{*}{$\mathrm{X}$} & \multirow{5}{*}{-} & Comercial & - & $\mathrm{X}$ & \\
\hline & & & Industrial & - & $\mathrm{X}$ & \\
\hline & & & \multirow{3}{*}{ Residencial } & $\begin{array}{l}\text { Condomínio } \\
\text { horizontal }\end{array}$ & - & $\mathrm{X}$ \\
\hline & & & & $\begin{array}{c}\text { Condomínio } \\
\text { vertical }\end{array}$ & - & $\mathrm{X}$ \\
\hline & & & & Ruas e Avenidas & $\mathrm{X}$ & - \\
\hline \multirow{5}{*}{ Grande } & \multirow{5}{*}{$\mathrm{X}$} & \multirow{5}{*}{ - } & Comercial & - & $\mathrm{X}$ & - \\
\hline & & & Industrial & - & $\mathrm{X}$ & - \\
\hline & & & \multirow{3}{*}{ Residência } & $\begin{array}{l}\text { Condomínio } \\
\text { Horizontal }\end{array}$ & $\mathrm{X}$ & - \\
\hline & & & & $\begin{array}{c}\text { Condomínio } \\
\text { Vertical }\end{array}$ & $\mathrm{X}$ & - \\
\hline & & & & Ruas e Avenidas & $\mathrm{X}$ & - \\
\hline
\end{tabular}

Fonte: Dados da pesquisa (2021).

A medida em que os municípios vão se tornando maiores em área, o planejamento urbano atende as maiores condições de funcionalidade, e, os dados dos trabalhos a partir do uso de sensoriamento para uso e ocupação dos solos urbanos lhes são úteis, uma vez que foram utilizados para a elaboração do planejamento.

Os municípios de pequeno porte, não aparecem como usuários de dados de sensoriamento remoto para planejamento urbano de uso e ocupação do solo, também não foi encontrado nesse trabalho nenhum indicio de planejamento urbano para esses municípios os de médio porte, considerados assim aqueles com mais de 50 (cinquenta) mil habitantes surgem como usuários desses dados para planejamento de áreas comerciais, industriais e residenciais para ruas e avenidas, já os de grande porte com mais de 100 (cem) mil habitantes, surgem como utilizadores dos dados de sensoriamento remoto para uso e ocupação de áreas comerciais, indústrias e residenciais em totalidade a depender da região geográfica de pertencimento do município, (Estatuto das Cidades, 2009).

Em se tratando de regiões geográficas, a região Sudeste lidera em número de produções científicas para o uso e ocupação do solo urbano a partir de dados de sensoriamento remoto, seguida pela região Sul, Nordeste, Centro Oeste e Norte, nesse sequência (ver Tabela 3); de todos os artigos analisados fica claro o uso desses dados para o planejamento urbano sendo destaque para os municípios do Rio de Janeiro e Monte Claros no Estado de Minas Gerais, ambos municípios da região Sudeste, nas demais regiões, as produções não deixam claro se os fazedores de políticas públicas para uso e ocupação dos solos urbanos fazem uso desses recursos para elaboração de suas políticas.

Há um consenso nos dados das produções em relação a importância do uso desses no planejamento urbano, levando ao entendimento de que ao utilizarem esses dados os fazedores dessas políticas tem maior probabilidade de acerto quanto a localização e distribuição dos solos urbanos na busca pela eficiência e respeito ao meio ambiente, possibilitando inclusive por características do uso e dos impactos desse uso os bairros por residências, comércio e indústria, tornando essas áreas mais produtivas e, consequentemente mais desenvolvida, nesse sentido, há uma propensão a concluir que regiões que mais fazem uso desses dados para planejarem a distribuição de seus espaços tornam-se mais desenvolvidas.

Em se tratando da distribuição das produções cientificas por regiões geográficas brasileira temos a seguinte distribuição por ordem numérica de produções, sudeste liderando com 15(quinze) produções, a Região Sul com 13(treze), 
Nordeste com 12(doze), Centro Oeste com 09 (nove) e Norte com 05(cinco) somando em conjunto um total de 54(cinquenta e quatro) trabalhos demonstrando o uso de dados de sensoriamento remoto para estudos de solos urbanos. Ver Tabela 3.

Tabela 3 - Distribuição de trabalhos científicos com dados de Sensoriamento Remoto para estudos de solos urbanos por regiões geográficas

\begin{tabular}{c|c}
\hline Região Brasileira & Quantidade de produções \\
\hline Norte & 05 \\
\hline Nordeste & 12 \\
\hline Centro Oeste & 09 \\
\hline Sudeste & 15 \\
\hline Sul & 13 \\
\hline Total & 54 \\
\hline
\end{tabular}

Fonte: Dados da pesquisa (2021).

Como dito acima, as Regiões com maior número de produção cientificas com dados de sensoriamento remoto são ao mesmo tempo as regiões que fazem uso dessas informações para planejar o uso e a ocupação de seus espaços urbanos, são essas regiões onde se encontram significativas universidades com produção do conhecimento com excelência.

A distribuição dessas produções por Regiões brasileiras é demonstrado no Gráfico 5, no qual demonstra que há uma maior representatividade dessas produções na Região Sudeste, Sul e Nordeste, e menos representativa no Centro Oeste e Norte, destas como dito acima, há evidencia de uso desses dados para o planejamento urbano com finalidades diversas e análise da situação urbana apenas na região Sudeste; nas demais ocorre a evidência de uso, mas não fica claro esse uso para tal fim nas produções analisadas.

Gráfico 5 - Distribuição por Regiões Geográficas Brasileiras da utilização dos dados do sensoriamento remoto em solos urbanos.

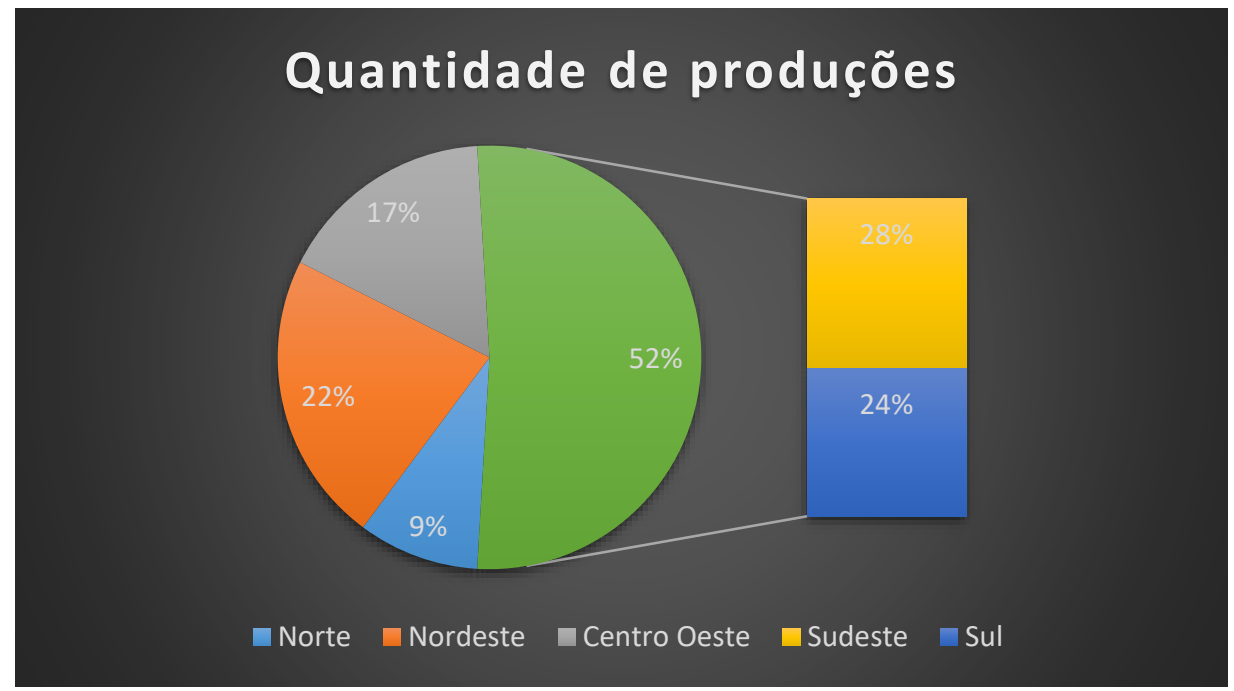

Fonte: Dados da pesquisa (2021). 
Quando da utilização para planejamento urbano tem-se a alocação organizada do espaço para instalação de comércio, de indústria e de residências. Essa última se subdivide e três categorias: condomínios horizontais, condomínios verticais e ruas e avenidas, seja qual for a finalidade quando o planejamento é feito a partir de dados de Sensoriamento Remoto, melhor tornase a utilização do espaço, por exemplo de Montes Claros em Minas Gerais, que ao utilizar dados de sensoriamento remoto para mapeamento do espaço urbano, identificando as áreas possibilitou a classificação por faixa de renda da população a ocupação em áreas mais pobres 'favelas' assim como bairros com maiores caractersiticas organizacionais, após o mapeamento os gestores passaram a usar a tecnologia para monitoramento das ações, avaliando a eficácia do planejamento (Leite \& Brito, 2011).

Quando a utilização é para outras finalidades, identificação de endemias, ilhas de calor, etc., nessas está incluso análise da situação dos condomínios urbanos, se devidamente ocupado ou se apenas com área delimitada e vendidas exclusivamente pela especulação financeira, análise da mobilidade urbana identificação de áreas baldias, etc., isto é, os planejadores são indicados nos trabalhos como utilizadores dos dados do sensoriamento remoto para identificar e entender problemas urbanos oriundos da ocupação do solo, mas não os usa efetivamente para a realização do planejamento para a ocupação desses espaços ao menos não fica clara essa utilização, (Amani-Beni, et. al., 2018).

Em se tratando do uso desses dados para análise dos espaços urbanos tem-se uma estrutura de organização na qual além da identificação desses espaços como descrito, tem-se as ditas projeções para uso das escolas, das praças públicas, do comércio e das residências de modo geral, e, essa análise parte para média de casos em que a utilização de dados de Sensoriamento Remoto sejam capazes de auxiliar na previsão de enchentes, deslizamentos, mobilidade social etc. de sorte que o uso de dados de sensoriamento para planejamento dos espaços urbanos, torna esse planejamento mais eficiente, com capacidade palpáveis de evitar sinistros e melhor alocar os recursos naturais disponíveis (Muahorea, et. al., 2017)

\section{Considerações Finais}

O Satélite Landsat5 foi o mais utilizado nas publicações com mais de cinco anos, nas publicações com menos de cinco anos o Landsat7 lidera as publicações, o Ikonos e o Quick Bird surgem de forma modesta, porém com o lançamento de satélites com novos sensores, surge o satélite Landsat7, capaz de ampliar as possibilidades de uso dos produtos Landsat, os demais são tendências que podem vir a liderar as produções a medida em que sua utilização seja uma realidade pelos profissionais da área, dito isto a partir do uso desses trabalhos em planejamento urbano em momentos recentes, realidade que não foi encontrada em publicações com mais de cinco anos.

Nas publicações com mais de cinco anos, praticamente não utilizamos sensoriamento remoto para o planejamento urbano, porém pode-se observar que nas publicações com até cinco anos era considerável.

O uso do planejamento urbano através do sensoriamento remoto nas cidades favorece a visão global dos problemas urbanos e a formulação de um modelo integrado para seu desenvolvimento futuro, porém pode-se constatar neste estudo que as cidades de pequeno porte não utilizam desta técnica, nas cidades de médio porte foi constatada a utilização apenas para uso comercial, industrial e residenciais para as ruas e avenidas, já nas cidades de grande porte surgem como utilizadores do sensoriamento remoto em todas às áreas do uso e ocupação do solo.

As Regiões Sudeste, Sul e Nordeste destacam-se com 74,07\% do total das produções cientificas pesquisadas em detrimento das regiões Norte e Centro Oeste que juntas somam 25,92\%, das produções cientificas, evidenciando que quanto mais desenvolvida a região maior será o uso de técnicas de sensoriamento remoto.

Assim pode-se concluir que a busca por meio de revisão bibliográfica sistemática, é possível identificar a situação do uso do SR e metodologias para o planejamento; nesse sentido, aconselha-se para trabalhos futuros utilizar essa ferramenta para observar os possíveis planejamentos para uso e ocupação de solos urbanos com vistas as cidades inteligentes, sustentáveis e 
viáveis do ponto de vista da produção de bens serviços e mercadorias da circulação dessas e, sem esquecer as pessoas, de sorte que possa se verificar se o uso do sensoriamento remoto e as produções cientificas realizadas a partir dessa ferramenta estão sendo utilizadas pelos tomadores de decisões e elaboradores de políticas públicas como instrumentos para construção de espaços urbanos mais harmoniosos com respeito ao meio ambiente e aos recursos naturais.

O aconselhamento acima, dar-se em função de que nesse trabalhos não foram abordados temas como sustentabilidade, cidades inteligentes, responsabilidade social e ambiental nem tampouco a viabilidade da produção de bens serviços e mercadorias e a circulação desses e das pessoas, fator que são interessantes de serem abordados em pesquisas futuras, pois no presente trabalhos abordou-se exclusivamente trabalhos que utilizassem do sensoriamento remoto para o planejamento do uso e ocupação dos solos urbanos compreendendo que esse planejamento amenizaria o processo de afavelamento comum nos centos urbanos nacional.

\section{Referências}

Almeida, C. M. de (2010). Aplicação dos sistemas de sensoriamento remoto por imagem e o planejamento urbano regional. USJT.ORQ.URB. no 3 primeiro semestre.

Amani-Beni, M. et. al. (2018). Impact of urban parks tree, grass and water body on microclimate in hot summer days: a case study of Olympic Park in Beijing, China. Urban Forestry \& Urban Greening, 32, 1-6.

Anderson, J. R. et al. (1976). A land use and land cover classification. System for use with remote sensor date. Geological Survey Profissional paper 964. 41p. http://landcover.ugs.gov/pdf/Anderson.pdf .

Anderson, P. S. (1982). Fundamentos para fotointerpretação/Paul S. Anderson, editor coordenador. Rio de Janeiro: Sociedade brasileira de cartografia.

Aquino, Í. de S. (2010). Como escrever artigos científicos: sem “arrodeios” e sem medo da ABNT/ Ítalo de Souza Aquino. Sariva.

Aragão, R., Cruz, M. A. S., Correia, E. C. O., Machado, L. F. M., \& Figueiredo, E. E. (2017). Impacto do uso do solo pelo aumento da densidade populacional sobre o escoamento numa área urbana do Nordeste Brasileiro via geotecnologias e modelagem hidrológica. Revista Brasileira de Geografia Física, v.10, n.02.

Barros, L. C. (2005). Análise da Expansão Urbana de Betim Através do Modelo de Mistura. In: VII Curso de Especialização em Geoprocessamento, 2005, Belo Horizonte. VII Curso de Especialização em Geoprocessamento, Belo Horizonte. 1-38. http://www.csr.ufmg.br/geoprocess amento/publicacoes/luizacaldeira.pdf.

Castro, A. W. S. (2000). Clima urbano e saúde: as patologias do aparelho respiratório associadas aos tipos de tempo no inverno, em Rio Claro - SP. 2000. 202p. Tese (Doutorado em Geografia) - Instituto de Geociências e Ciências Exatas, Universidade Estadual Paulista "Julio de Mesquita Filho", Rio Claro.

Costa, A. N., \& Alves, M. G. (2005). Monitoramento da expansão urbana no Município de Campos dos Goytacazes - RJ. In: SBSR, Simpósio Brasileiro de Sensoriamento Remoto. Goiânia.

EEA. European Environment Agency. (1990) Corine Land cover. Compenhagen, 94p.

Fitz, P. R. (2008). Geoprocessamento sem complicação / Paulo Roberto Fitz. Oficina de textos.

Florenzano, T. G. (2020). Imagens de satélite para estudos ambientais. oficina de textos, 97p.

Forster, B. C. (1994). An examination of some problems and solutions in urban monitoring from satellite platforms. International Journal of Remote Sensing, $6,139-151$.

Fuckner, M. A. (2006). Aplicações do Sensoriamento Remoto no Estudo do Crescimento Urbano. Universidade Federal de Santa Catarina - SC.

Gil, A. C. (2008). Métodos e técnicas de pesquisa social. (6a ed.), Editora: Atlas S/A.

Haas, A. et al. (2018). Delimitação e caracterização de app através do uso de um sistema de informação geográfica (SIG): o caso das APPs nos cursos de água da sub-bacia do Lajeado Pardo, noroeste do RS. Revista Gestão \& Sustentabilidade Ambiental, 7, 640-649.

Henriques C. D. (2008). Maputo, Cinco décadas de mudança territorial: o uso do solo observado por tecnologias de informação geográfica. Lisboa, cooperação portuguesa.

Jensen, John R. (2009). Sensoriamento remoto do ambiente: uma perspectiva em recursos terrestres / John R. Jensen: tradução José Carlos Neves Epiphanio (coordenador) ... Parêntese.

Landsberg, H. E. (1981). The urban climate. International Geophysics Series, 28. Academic Press. 275p.

Leite, M. E. \& Brito, J. L. S. (2011). Sensoriamento remoto aplicado ao mapeamento do uso do solo urbano e de assentamentos ilegais em Monte Carlos-MG. Geosul, 26, 99-128 
Miranda, Y. C. (2018). Sensoriamento remoto aplicado a detecção de ilhas de calor urbanos: uma revisão sistemática. UTFPR.

Monteiro, C. A de F. \& Mendonça, F. (2003). (Org.). Clima Urbano. Editora Contexto. 192p.

Mushorea, C. et al. (2017). Remote sensing applications in monitorin gurban growth impact sonin-and outdoor thermal conditions: a review. Remote Sensing Applications: Society and Environment, 8, 83-93.

Oraquine, O. A. (2020). Estimation de áreas agrícolas mediante classificacion basada em objetos can imagenes de satélité, en el municipio de Okinawa Dell Dpto de Santa Cruz. REVIGEO.

Panizza, A. de C. \& Fonseca, F. P. (2011). Técnicas de interpretação visual de imagens. GEOSUSP - Espaço e Tempo, São Paulo, nº $30,30-43$.

Pereria, J. F., Neves, E. \& Andrade, C. J. D. (2018). Metodologia da pesquisa cientifica.

Portis, G. T., Santos, A. M. dos \& Nunes, F. G. (2020) Análise espaço temporal da alteração do uso do solo sob influência de um polo gerador de viagens em Goiânia, GO, Brasil. Ambiente Construído. 20, 513-525.

Santos, J. S. M. \& Lapolli, F. M. (2003) Comparação tabular da expansão urbana dos municípios de Itapema, Pato Belo e Bombinhas em Santa Catarina, Brasil, no período de 1985 z 2002. Universidade Federal de Santa Catarina.

Tavares, A. C. \& Prochnow, C. A. C. (1997). Atlas climático de Rio Claro - SP. (s.n.). 78p.

Xinmin, W., Jing, L. \& Hongyang, Y. (2021). An improved anti-quantium MSTS public Key encryption scheme for remote sensing images. Interprise information systems. 15, 530-544 SI, APR.

Zhongping, S., et al. (2013). Estimation of net primary productivity of Sanhu national nature reserve in China by integrating HJ-1 Remote sensing image with surface energy balance algorithm for Land Model. Sensor Latter, 11, 1121-1127

Zhou, D. et al. (2019). Satellite remote sensing of surface urban heatis lands: progress, challenges, and perspectives. Remote Sensing, 11, 2-36. 